of lymphocytes selectively stimulated to proliferate after removal of IgGs from the circulation.

We did not expect the rapidity with which the pretibial myxoedema regressed in this patient. A plasma factor, possibly another IgG, may be responsible for the induction of pretibial myxoedema. Sera from patients with pretibial myxoedema enhance fibroblast growth and proliferation in vitro ${ }^{18}$ and accelerate the incorporation of labelled glucosaminoglycan precursors by fibroblasts in culture. ${ }^{19}$ The remarkably rapid resolution and the equally rapid reappearance of pretibial myxoedema in this patient indicate an extremely rapid turnover of glycosaminoglycans.

We suggest that plasmapheresis has a useful place in the treatment of the ophthalmopathy and pretibial myxoedema of Graves's disease. Both these complications have been extremely refractory to any form of treatment. The choice of patients must be made with care, as it is only in the acute and rapidly progressive disease that improvement is likely to occur.

We thank Professors Deborah Doniach and Ivan Roitt for their critical comments and help in the preparation of this paper, Professor A V Hoffbrand and Dr J Moorhead for the use of the Hemonetics cell separator, and Mrs M Elleman for secretarial help. NJM and SPB are grateful to the MRC for support during the course of this work.

\section{References}

${ }^{1}$ Smith, B R, et al, fournal of Endocrinology, 1977, 75, 401.

2 Doniach, D, and Marshall, N J, in Autoimmunity, ed N Talal. New York, Academic Press, 1977.

${ }^{3}$ Schleusener, H, et al, fournal of Endocrinological Investigation, 1978, 2, 155.

${ }^{4}$ Brown, R S, et al, Lancet, 1978, 1, 904.

${ }^{5}$ Mukhtar, E D, et al, Lancet, 1975, 1, 713

${ }^{6}$ Dandona, P, and El Kabir, D J, Clinical Science and Molecular Medicine, $1970,38,2 \mathrm{P}$.

' Winand, R J, Salmon, J, and Lambert, P, Wiener Medizinischen Akademie, 1971, p. 583.

${ }^{8}$ Singh, S P, and MacKenzie, J M, Metabolism, 1971, 20, 49F.

9 Winand, R J, and Kohn, L D, Proceedings of the National Academy of Sciences, 1972, 69, 1711 .

${ }^{10}$ Dandona, P, D Phil thesis, University of Oxford, 1974.

${ }^{11}$ Bolonkin, D, et al, fournal of Biological Chemistry, 1975, 250, 6516.

${ }^{12}$ Lockwood, C M, Rees, A J, and Pearson, T A, Lancet, 1975, 1, 711.

${ }^{13}$ Pinching, A J, Peters, D K, and Newson-Davis, J, Lancet, 1976, 2, 1373.

${ }^{14}$ Dau, P C, et al, New England fournal of Medicine, 1978, 297, 1134.

${ }^{15}$ Gill, D L, Marshall, N J, and Ekins, R P, Molecular and Cellular Endocrinology, 1978, 10, 89 .

${ }^{16}$ Bystryn, J C, Graf, M W, and Uhr, J W, fournal of Experimental Medicine, 1970, 132, 1279.

${ }^{17}$ Branda, R F, Moldow, C F, and McCullough, J J, Transfusion, 1975, 15, 570.

${ }^{18}$ Cheung, H S, et al, Clinical Research, 1977, 125, 147A.

${ }^{19} \mathrm{Jolliffe}, \mathrm{D}$, personal communication, 1978.

(Accepted 22 November 1978)

\title{
Treatment of irritable bowel syndrome with lorazepam, hyoscine butylbromide, and ispaghula husk
}

\author{
J A RITCHIE，S C TRUELOVE
}

British Medical fournal, 1979, 1, 376-378

be more effective, but it will be possible to determine this only by carrying out factorial therapeutic trials.

\section{Summary and conclusions}

A double-blind controlled therapeutic trial of factorial design was used to study the therapeutic effects of lorazepam, hyoscine butylbromide, and ispaghula husk in 12 randomised blocks of eight patients with the irritable bowel syndrome (IBS). Each of the three agents caused a sustained symptomatic improvement in some of the patients, although only with ispaghula was the difference between the real and dummy preparation statistically significant. When the eight possible combinations of treatment were analysed none of the 12 patients who received only dummy preparations of the three agents had maintained any improvement over the three months of the trial. Seven patients improved among the 12 who received potent preparations of all three agents, and between four and six patients improved in the groups receiving one or two of the potent preparations.

These therapeutic results, though far from perfect, show that the types of drug commonly used to treat IBS are of some value and may be additive in their effects. Similar combinations of other therapeutic agents may

\footnotetext{
Nuffield Department of Clinical Medicine, Radcliffe Infirmary, Oxford

J A RITCHIE, MA, DM, research fellow

S C TRUELOVE, MD, FRCP, consultant physician
}

Therove, Mo, kncr, consultant physician

\section{Introduction}

The irritable bowel syndrome (IBS) is a diagnostic label applied to patients who have various gastrointestinal symptoms that cannot be attributed to any recognised pathological process. These symptoms commonly include a disturbance of bowel function, with diarrhoea or constipation (or an alternation of the two), hypersecretion of colonic mucus, gastrointestinal flatulence, abdominal pain, and a general lack of subjective wellbeing expressed variously as tiredness, weakness, or depression. These symptoms may be either continuous or episodic. They appear to be related to excessive motor activity of some part of the gut, possibly through hyperreaction to parasympathetic and hormonal stimuli. Emotional tensions are apt to precipitate or exacerbate an episode of symptoms. It is a common condition and in a small proportion of those affected may give rise to severe disability. For many years medical treatment has been based on one or more of three types of therapeutic agent, with additional symptomatic control by aperients or antidiarrhoeal drugs as necessary. The three types of agent are: (1) an antispasmodic drug, usually either an anticholinergic or mebeverine; (2) a sedative or tranquilliser, originally phenobarbitone but now commonly a benzodiazepine derivative; and (3) an inert faecal bulking agent, either pharmaceutical or obtained by way of increased dietary roughage.

The present study was undertaken to test the validity of clinical impressions that the basic treatment with antispasmodic, tranquilliser, and bulk-provider is of value in IBS. 


\section{Methods}

\section{CHOICE OF THERAPEUTIC AGENTS}

The three therapeutic substances that were chosen for the test were hyoscine butylbromide (Buscopan) in a dose of $10 \mathrm{mg}$ four times a day, lorazepam (Ativan) $1 \mathrm{mg}$ twice a day, and an ispaghula derivative (Fybogel) one sachet twice daily. Choice depended in part on the ability of the makers to provide a dummy preparation with identical physical characteristics. Hyoscine butylbromide by intravenous injection is effective in relieving gastrointestinal spasm in the course of radiological and endoscopic examinations. Lorazepam has a longer duration of action than other benzodiazepine tranquillisers and in ordinary clinical practice appears to be effective with only two doses a day. Bran would have been a logical choice as a bulk provider but no one has yet suggested a convincing fibre-free dummy preparation, so ispaghula husk was used.

\section{DESIGN OF THE TRIAL}

To test three therapeutic substances simultaneously against their corresponding dummy preparations, singly and in combination, a trial with a factorial design must be used. In the present study two combinations, real or dummy, were possible for each of the three therapeutic agents to be tested, which meant that there were $2^{3}=8$ possible combinations of treatment. If $A$ represents real and a dummy lorazepam, B real and b dummy hyoscine butylbromide, and $\mathrm{F}$ real and $f$ dummy ispaghula husk, then the eight possible combinations are $\mathrm{ABF}, \mathrm{ABf}, \mathrm{aBF}, \mathrm{AbF}, \mathrm{Abf}, \mathrm{aBf}, \mathrm{abF}$, and abf. The patients were all numbered consecutively, and each block of eight patients received all eight combinations of the three treatments, which were issued in random order by the hospital pharmacist.

Within each of the "randomised blocks" each of the three agents was taken by four patients, and each dummy preparation by the other four. For example, the four patients who received real lorazepam (A) were those who were issued with $\mathrm{ABF}, \mathrm{ABf}, \mathrm{AbF}$, and $\mathrm{Abf}$, while those who received dummy lorazepam (a) instead got $a B F, a B f, a b F$, and abf. Thus the four subjects who made up each half-block received, as well as either $A$ or a, the same four additional treatment combinations-namely, $\mathrm{BF}, \mathrm{Bf}, \mathrm{bF}$, or bf-in exact balance. This means that any difference that occurred in the improvement scores between patients with IBS who were treated with lorazepam and those who were given dummy lorazepam could be attributed solely to the real agent. Similarly, half the members of the randomised block received hyoscine butylbromide $(B)$ and the other half the dummy preparation (b) in addition, in each case, to AF, Af, aF, or af. Once again, the treatments allocated to the two half-blocks differed only in respect of B or b. Similarly, four members of the eight-patient block were treated with ispaghula husk (F) and four given the dummy preparation (f). A total of 96 patients were brought into the present trial, making up 12 randomised blocks.

\section{ASSESSMENT OF RESULTS}

Not only do the symptoms of IBS vary widely between one patient and another, but the patients' reactions to them may also differ. For example, one patient may find pain the most distressing feature, another the diarrhoea, and yet another the subjective disability of weakness, lethargy, and depression. In the absence of a specific cure the objective of treatment must be to obtain an overall symptomatic improvement and not necessarily to eliminate individual symptoms. Assessment of the value of treatment was therefore based on the patients' clearcut awareness of symptomatic improvement, provided that this did not conflict with the clinical impression of the observer. No attempt was made to assess the degree of improvement in each symptom after treatment. Three grades were originally proposed for the assessment - namely, symptom free, improved, and unchanged or worse. Only five patients, however, became entirely symptom free and remained so throughout the whole trial period. This meant that the only two grades of response that were of any real value were improved and not improved.

The duration of the trial is crucial in assessing treatment in IBS. Patients with IBS often seem to be improved over the course of a few weeks with any new regimen or with the fresh interest of being entered in a therapeutic trial, only to relapse when the novelty has worn off. To obviate this defect within practicable limits we assessed progress after four weeks and again after three months. Only those patients who thought that they were better at both assessment sessions were counted as improved.

One patient dropped out of the trial before the first assessment, and the pharmacist reallocated her treatment combination to the next newcomer. Three patients dropped out or had to be taken out after their first assessment, at which they had reported an improvement. In these cases the treatment combination was again reallocated to a later patient number.

\section{PATIENTS}

All the 96 patients who completed the trial had been fully investigated in a specialist gastroenterological outpatient clinic; this investigation had not elicited evidence of any organic disease likely to be causing their symptoms. Their ages ranged between 16 and 69 (mean 38 ) years, and $74(77 \%)$ were women.

\section{Results}

Of the 96 patients taking part in the trial, only $36(38 \%)$ reported an improvement in their symptoms and maintained it over the three months of the trial. Had the study been terminated at four weeks the proportion would have been $49 \%$ and this would have included three out of 12 abf controls, whereas at the end of three months none of the patients in this group showed any sustained improvement.

Effect of individual agents-Table I shows the therapeutic effectiveness of each of the three agents tested. Patients who received any one of them were more likely to experience sustained improvement than those who did not. A significantly increased probability, however, was obtained only with ispaghula husk $\left(\chi^{2}=4.44 ; \mathrm{P}<0.05\right)$.

TABLE I-Therapeutic effectiveness of lorazepam, hyoscine butylbromide, and ispaghula husk compared with dummy preparations in the 48 patients who received each agent or its dummy form

\begin{tabular}{|c|c|c|c|c|c|c|}
\hline & \multicolumn{2}{|c|}{ Lorazepam } & \multicolumn{2}{|c|}{$\begin{array}{c}\text { Hyoscine } \\
\text { butylbromide }\end{array}$} & \multicolumn{2}{|c|}{ Ispaghula husk } \\
\hline & Real & $\overline{\text { Dummy }}$ & Real & Dummy & Real & Dummy \\
\hline No $\left({ }^{\prime \prime}{ }_{1}\right)$ improved & $21(44)$ & $15(31)$ & $22(46)$ & $14(29)$ & $23(48)^{*}$ & $13(27)^{*}$ \\
\hline
\end{tabular}

*Significance of difference: $\chi^{2}=4 \cdot 44 ; P<0 \cdot 05$.

Effect of combinations of agents-Table II shows the actual numbers of patients in each of the eight 12-patient treatment groups who obtained benefit from the different combinations of agents. Among those who received lorazepam or hyoscine butylbromide alone (Abf or aBf) four were improved in each group. This was a better result than that obtained with dummy treatment (abf), but the difference was not significant $\left(\chi^{2}=2.7 ; P>0.05\right)$. Five of the 12 patients who received ispaghula husk alone $(\mathrm{abF})$ maintained their improvement over the whole three months, which was significantly different from the result obtained with dummy treatment (abf) $\left(\chi^{2}=4.0 ; \mathrm{P}<0.05\right)$. Five patients who received lorazepam and hyoscine butylbromide (ABf) and five who received lorazepam and ispaghula husk (AbF) also experienced sustained improvement, and the combination of hyoscine butylbromide and ispaghula husk (aBF) helped six of the 12 patients who took it $(P<0.02)$. Finally, of those who received the full treatment (ABF), seven maintained improvement over the three months $(\mathrm{P}<0 \cdot 005)$, showing a degree of additive effect between the three component agents.

TABLE II-Numbers of patients with irritable bowel syndrome who obtained benefit from aifferent combinations of lorazepam, hyoscine butylbromide, and ispaghula husk and dummy preparations

\begin{tabular}{lcccccccc}
\hline Treatment combination: & ABF & ABf & AbF & aBF & Abf & aBf & abF & abf \\
\hline No of patients improved & 7 & 5 & 5 & 6 & 4 & 4 & 5 & \\
No of patients not improved & 5 & 7 & 7 & 6 & 8 & 8 & 7 & 12
\end{tabular}
$A=$ Real lorazepam. $B=$ Real hyoscine butylbromide. $F=$ Real ispaghula husk
$a=$ Dummy lorazepam. $\mathrm{b}=$ Dummy hyoscine butylbromide. $\mathrm{f}=$ Dummy ispaghula $\mathrm{a}=\mathrm{B}$.
husk. 


\section{Discussion}

These results show, firstly, that each of the three components of the standard treatment for IBS-that is, the tranquilliser, anticholinergic, and bland bulk-provider-is more effective as treatment than the corresponding placebo; secondly, a combination of two agents tends to be more helpful than a single agent; and, thirdly, the use of all three agents together is better than any two.

The apparent therapeutic value of placebo in some controlled studies of treatment of IBS is largely inversely proportional to the duration of the trial. Over a short period, as in the threeweek trial by $\mathrm{McHardy}$ et $a^{\mathrm{l}}$ of chlordiazepoxide with clidinium bromide, up to $35 \%$ of patients may be relieved by a placebo. This is comparable with the improvement shown in the present study after four weeks by three of the 12 patients who received the dummy preparations (abf). In a trial lasting two or three months it is not unreasonable for no patients to improve with placebo, as Kleckner" found in his evaluation of mepenzolate bromide, though this prompted Ivey $^{3}$ to reject his findings without further consideration. Rhodes et al, ${ }^{4}$ testing phenobarbitone, belladonna, and placebo, found only one patient out of 15 who showed a preference for placebo compared with seven for each of the two active drugs. All the evidence thus suggests that patients with IBS derive little benefit from placebo treatment.

Although results of conventional medical treatment are better than those of placebo treatment, they are far from perfect. Even the most effective combination tested in the present studynamely, lorazepam, hyoscine butylbromide, and ispaghula husk -resulted in improvement in only seven out of 12 patients receiving it. Some other combination of therapeutic agents, however, might possibly be much more effective. For example, it is not only scientifically but also economically important to find out whether ordinary wheat bran is an effective substitute for ispaghula husk. Mebeverine may be better than an anticholinergic drug, and an antidepressive better than a tranquilliser. These and other combinations of therapeutic agents need to be tested in IBS, and we intend to set up further trials for this purpose.

Meanwhile we conclude that a combination of a tranquilliser, antispasmodic, and bland bulk-provider is more effective in treating IBS than any single agent or pair of agents. A physician should be prepared to try various alternatives within these three types of therapeutic agents if a patient does not show a good symptomatic response to the first combination.

This therapeutic trial was supported by a project grant from the Medical Research Council.

\section{References}

${ }^{1}$ McHardy, G, et al, Gastroenterology, 1968, 54, 508.

${ }^{2}$ Kleckner, M S, American fournal of Gastroenterology, 1959, 5, 609.

${ }^{3}$ Ivey, K J, Gastroenterology, 1975, 68, 1300.

${ }^{4}$ Rhodes, J B, Abrams, J H, and Manning, R T, Gastroenterology, 1973, 64, 829.

(Accepted 29 November 1978)

\title{
Mianserin and agranulocytosis
}

\author{
D A CURSON, A S HALE
}

British Medical fournal, 1979, 1, 378-379

\section{Summary and conclusions}

An alcoholic woman who was admitted to hospital for detoxification was prescribed thyroxine because of hypothyroidism and mianserin to alleviate severe depression. After several weeks' treatment she became unwell and was readmitted to hospital. Haematological examination indicated agranulocytosis. Further extensive investigations elicited no cause for this other than the mianserin, since no such disturbance has been reported for thyroxine after years of use.

Thus mianserin is probably implicated in this case of agranulocytosis. Although the response may have been idiosyncratic, it highlights the need to monitor new drugs during the early phases of widespread use.

Department of Psychiatry, Guy's Hospital, London SE1

D A CURSON, MRCPSYCH, DPM, senior registrar (present appointment: consultant psychiatrist, St Andrew's Hospital, Northampton)

A S HALE, BSC, medical student

\section{Introduction}

We report a case of agranulocytosis associated with treatment with the antidepressant mianserin. We believe that this is the first case to be described.

\section{Case report}

A 49-year-old alcoholic woman was admitted to hospital in December 1977 for detoxification. She was found to have hypothyroidism and was prescribed thyroxine $0.1 \mathrm{mg}$ daily before discharge two weeks later. She was readmitted on 14 February 1978 for further detoxification and psychiatric assessment of her depressive state and suicidal ideation. A blood picture was normal. She remained severely depressed, however, and mianserin $20 \mathrm{mg}$ three times daily was started on 23 February. She made an excellent recovery and was free of all depressive symptoms three weeks later.

On $28 \mathrm{March}$, during the fifth week of mianserin treatment, she returned to hospital from weekend leave to report that she felt generally unwell and had a sore throat. On examination she was pyrexial (temperature $38.2 \mathrm{C}$ ), her throat was inflamed, and she had cervical lymphadenopathy. Her only medication had been the thyroxine and mianserin at the prescribed doses. Two full blood pictures obtained on that same day showed a complete absence of neutrophils. She was transferred to a medical ward, the mianserin was stopped, and she was managed with reverse-barrier nursing, gut sterilisation, and systemic antibiotics. A sternal marrow biopsy on 31 March disclosed an $\mathrm{L}: \mathrm{E}$ ratio of $1: 1$. Erythropoiesis was active and normoblastic. 